\title{
A Phase 1b Trial to Assess the Pharmacokinetics of Ezutromid In Pediatric Duchenne Muscular \\ Dystrophy Patients on a Balanced Diet
}

Francesco Muntoni ${ }^{1}$, Bina Tejura ${ }^{2}$, Stefan Spinty $^{3}$, Helen Roper ${ }^{4}$, Imelda Hughes ${ }^{5}$, Gary Layton ${ }^{6}$ Kay E.

Davies $^{7}$, Shawn Harriman ${ }^{2}$ and Jonathon Tinsley ${ }^{2}$

${ }^{1}$ Dubowitz Neuromuscular Centre, University College London Institute of Child Health, London, UK;

${ }^{2}$ Summit Therapeutics, Abingdon, UK;

${ }^{3}$ Alder Hey Children's NHS Foundation Trust, Liverpool, UK;

${ }^{4}$ Birmingham Heartlands Hospital, Heart of England NHS Foundation Trust, Birmingham, UK;

${ }^{5}$ Royal Manchester Children's Hospital, Central Manchester University Hospitals NHS Foundation Trust, $U K$;

${ }^{6}$ ParamStat Limited, Ash, UK;

${ }^{7}$ MRC Functional Genomics Unit, University of Oxford, Department of Physiology Anatomy and Genetics, Oxford, $U K$

8. NIHR Great Ormond Street Hospital Biomedical Research Centre, 30 Guilford Street, London WC1N IEH, UK.

\section{Corresponding author:}

Professor Francesco Muntoni

Dubowitz Neuromuscular Centre

UCL Institute of Child Health

30 Guilford Street,

London WC1N 1EH, UK

Tel: 00442079052111

Fax: 00442079052832

Email: f.muntoni@ucl.ac.uk

\section{Disclosure Statement}

JT, BT, and SH are/were employees of Summit Therapeutics. NR and GL are paid consultants to Summit Therapeutics. KED is a shareholder in Summit Therapeutics. FM was involved as a Chief Investigator in a Summit clinical trial of ezutromid. He is also Chief Investigator of a Sarepta sponsored clinical trial; and Principal investigator of Ionis Pharmaceuticals / Biogen; Roche; PTC Therapeutics and Prosensa / Biomarin sponsored clinical trials. FM is a current member of the Pfizer Rare Disease Scientific Advisory Board and has provided ad hoc consultancies for Nicox; Italfarmaco; Akashi Therapeutics, Summit Therapeutics, Roche and PTC Therapeutics. HR is/was involved as a principal investigator (PI) in two Summit clinic trials of ezutromid and is a PI in clinical trials sponsored by Roche and a member of the DMB for Biomarin/Prosensa sponsored trials. HR has acted as a consultant for Biomarin and Novartis.

\section{Word count}

Abstract: 243

Body text: 4353

Figures/Tables: 4/5 


\title{
References: 7
}

Keywords: Utrophin, Duchene muscular dystrophy (DMD), food, pharmacokinetics, Phase 1b Running title: Phase $1 \mathrm{~b}$ Randomized Study in DMD patients

\begin{abstract}
Ezutromid (SMT C1100) is a small-molecule utrophin modulator which was being developed to treat Duchenne muscular dystrophy (DMD). Previous clinical trials of this agent revealed lower exposure in DMD patients compared with healthy volunteers which may reflect differences in diet. This study evaluated the pharmacokinetics of ezutromid in patients with DMD who followed a balanced diet. This was a multicenter, double-blind, placebo-controlled, ascending single and multiple oral dose study. Twelve pediatric patients were randomly allocated to one of three treatment sequences within which were three-treatment periods of 2weeks each. Each patient received, in a dose-escalating fashion, $1250 \mathrm{mg}$ and $2500 \mathrm{mg}$ BID of ezutromid administered orally as a micro-fluidized suspension (F3) with placebo in the other treatment period. Throughout the study, patients followed a balanced diet including recommended proportions of major food groups and administration of drug accompanied with $100 \mathrm{~mL}$ full-fat milk. This approach improved the absorption of ezutromid resulting in higher systemic exposure, with considerable variability in exposure between patients at each dose level. Single and multiple oral doses of $1250 \mathrm{mg}$ and $2500 \mathrm{mg}$ BID were considered safe and well tolerated. No severe or serious adverse events (AEs) and no study discontinuations due to AEs were reported. This study provides assurance that, with the formulation tested (F3) and instructions regarding food (balanced diet and whole fat milk), $2500 \mathrm{mg}$ BID ezutromid achieves plasma concentrations that - based on preclinical studies - should be able to modulate utrophin expression in future clinical trials.
\end{abstract}




\section{Introduction}

Duchenne muscular dystrophy (DMD) is an X-linked recessive disease caused by mutations in the dystrophin gene that results in skeletal, respiratory and cardiac muscle weakness. ${ }^{1}$ The disease progresses with loss of respiratory and cardiac function and is ultimately fatal. ${ }^{2}$ Utrophin is an autosomally-encoded homologue of dystrophin and has a similar function to dystrophin. ${ }^{3}$ It is present during both fetal development and muscle regeneration but is downregulated and subject to strict spatial restriction in mature fibers, where dystrophin expression normally predominates. Replacement of missing dystrophin with utrophin is a potential therapeutic strategy for DMD. Ezutromid (2-arylbenzoxazole [5-(ethylsulfonyl)-2(naphthalen-2-yl) benzo[d]oxazole]; formerly SMT C1100) is a small molecule utrophin modulator ${ }^{4}$ (Figure 1, Panel A). In vitro studies show that levels of utrophin RNA and utrophin protein are increased in human muscle cells following application of ezutromid. ${ }^{5}$

In vivo data from a mouse DMD model indicated that dosing over a period of 28 days led to an increase in utrophin mRNA and protein levels compared with vehicle controls. ${ }^{5}$ The increase in total utrophin was due to more fibers being utrophin positive rather than increases in utrophin in already positive fibers. Utrophin levels were increased in heart muscle and the diaphragm and improvement in exercise and capacity were observed in these studies. In vitro studies in human and DMD myoblasts and myotubes treated with the utrophin modulator, ezutromid (in the presence of protein) followed a concentration-response curve with respect to utrophin protein with an estimated $\mathrm{EC}_{30}$ of $34 \mathrm{ng} / \mathrm{mL}(\sim 0.1 \mu \mathrm{M})$ and $\mathrm{EC}_{50}$ of $67 \mathrm{ng} / \mathrm{mL}(\sim 0.2 \mu \mathrm{M})^{6}$. In in vivo efficacy studies, using dystrophin deficient $m d x$ mice, a single $50 \mathrm{mg} / \mathrm{kg}$ daily dose achieved levels above $30 \mathrm{ng} / \mathrm{ml}$ for several hours in a 24 -hour period resulting in a significant reduction in the muscle pathology which characterises this model. Although the therapeutic target exposures in the systemic circulation in the human are not known for ezutromid, early clinical studies have been designed to achieve exposures in excess of $30 \mathrm{ng} / \mathrm{mL}$ based on the preclinical data. As the clinical data emerge, the exposure response relationship between the target engagement biomarker (utrophin expression) and disease biomarkers (e.g. developmental myosin positive fibers, muscle fat fraction) will be explored to determine the most appropriate therapeutic target and exposure metrics. 
Ezutromid is a Biopharmaceutics Classification System (BCS) class II compound and is highly insoluble in water; it is also highly protein bound across species (humans >98\%). Cytochrome (CYP) P450 profiling suggests that CYP1A1 and CYP1A2 are primarily responsible for its primary metabolism, with evidence of subsequent glucuronidation. In human liver microsomes, there was no evidence of inhibition of any of the major CYP450s; however, one of the major metabolites has the potential to inhibit CYP2B6 (Summit, data on file). The pharmacokinetic properties of ezutromid have been determined in two prior clinical studies. In patients, ezutromid is rapidly absorbed and, after achieving peak concentration, generally shows a biphasic decline with a terminal half-life of between $5-10$ hours after single dose ${ }^{7}$. High between-subject variability and a positive food effect has previously been observed with ezutromid in both healthy volunteers ${ }^{6}$ and patients ${ }^{7}$. Ezutromid is metabolized to two major metabolites - dihydrodiol (DHD) I and dihydrodiol III (Figure 1, Panels B and C). Both metabolites have similar PK properties to the parent molecule but are markedly more abundant in plasma ${ }^{7}$. Both metabolites have also been shown to have some pharmacological activity in vitro; compared to ezutromid, DHD I has a 31-fold lower potency and DHD III has a potency that was 6.5-fold lower (Summit Therapeutics, data-on-file).

Following the first healthy volunteer study, a multicenter, Phase $1 \mathrm{~b}$ study in twelve DMD patients confirmed that ezutromid was safe and well tolerated; however, a high degree of inter-patient variability in ezutromid plasma concentrations was observed, with most patients experiencing much lower exposures than those expected based on the prior findings in healthy volunteers ${ }^{7}$. It was hypothesized that exposure differences between adult healthy volunteers and DMD patients could be due to the reduced absorption of ezutromid in patients adhering to low-fat diets. Indeed many patients with DMD follow reduced fat diets to counter potential weight gain associated with corticosteroid therapy (used as part of standard of care) and immobility (due to disease progression). The hypothesis was that dietary differences, as opposed to populations differences, were driving the lower exposure in DMD patients. 
The primary aim of the current study was to evaluate the pharmacokinetics of single and multiple oral doses of ezutromid in patients with DMD who follow a balanced diet; additional aims included further investigation of the drug's safety and tolerability in this context.

\section{Methods}

\section{Study Ethics}

The study protocol and informed consent form (ICF) were reviewed and approved by the centralised Ethics Committee (EC; NRES Committee London - West London \& GTAC). The study commenced after EC approval and receipt of a Clinical Trials Authorization from the Medicines and Healthcare Products Regulatory Agency (MHRA). Four sites (Great Ormond Street Hospital for Children; Alder Hey Children's Hospital; Birmingham Children's Hospital; Royal Manchester Children's Hospital) were initiated following approval from their respective Research \& Development (R\&D) committees (University College London, Institution of Child Health; Alder Hey Children's NHS Foundation Trust; Birmingham Children's Hospital NHS foundation Trust; Central Manchester University Hospitals NHS Foundation Trust).

A parent/legal guardian dated and signed the written consent on behalf of the patient, or, if age-appropriate, the patient provided consent or assent per ICH and local regulations. The studies were carried out in accordance with Good Clinical Practice, consistent with the Declaration of Helsinki. Clinical Trial Registry Number is NCT02383511.

\section{Study Design}

This was a multicenter, 3-period, 3-treatment, double blind, placebo controlled, ascending single and multiple oral dose study conducted in pediatric DMD patients. The expected duration of patient participation (from Screening to post study Follow-up Visit) was a minimum of 16 weeks.

\section{Study Population}

The study population comprised pediatric male patients, aged between 5 and 13 years (both inclusive) with a 
genetic diagnosis of DMD. Adherence to a balanced diet was required from one week prior to dosing until the end of the follow-up period. The patients needed to have six months or more of stable systemic corticosteroid therapy prior to screening; modification of steroid dose for body weight was permitted (as per standard of care). Patients were excluded if they had enrolled or participated in any therapeutic trial within the previous three months; had a known hypersensitivity to the excipients of the study drug or a previous history of drug allergy; were dairy or lactose intolerant; or had an allergy to egg or nuts or any other dietary restrictions that could interfere with the conduct of the study. Also excluded were patients taking a prohibited medication within five half-lives prior to baseline assessments including: inducers and moderate/strong inhibitors of CYP1A2, substrates for CYP1A2 with narrow therapeutic windows, nicotine, herbal supplements, and homeopathic preparations and inhibitors of breast cancer resistance proteins. Smoking, exposure to daily passive smoking and certain foods were prohibited to minimize environmental factors which may cause CYP1A induction. Non-ambulatory patients, those requiring mechanical ventilation and those with intermittent or continuous difficulties in swallowing were also excluded. Other exclusion criteria were: clinically significant acute illness within 4 weeks of the start of dose administration; a comorbidity that increased the risk of participating in the study; symptomatic cardiomyopathy; an abnormality in 12-lead ECG; any clinically significant medical condition, other than DMD, that might interfere with the interpretation of safety or pharmacokinetics (PK) evaluations.

\section{Study design}

The primary objective of the study was to evaluate the PK of ezutromid and its metabolites in patients with DMD who were following a balanced diet. Secondary objectives included assessment of safety and tolerability, evaluation of the diurnal variability in the steady-state PK of ezutromid and evaluation of creatine phosphokinase (CPK) as a potential pharmacodynamic biomarker.

Screening of patients was performed within 28 days prior to first dose. A physical examination was conducted as well as clinical laboratory evaluations and a 12-lead ECG. Body weight, blood pressure, pulse rate and body temperature were determined. Patients were registered into the study at the Screening visit via an 
interactive web response system (IWRS; Cenduit LLC) and assigned a unique patient identification number. Twelve patients were randomized to three treatment sequences with four patients per treatment sequence groups. Each patient received both doses of ezutromid (1250 mg BID and $2500 \mathrm{mg}$ BID) in a dose escalating fashion, with placebo in the other treatment period (Figure 2). Each treatment period consisted of a single dose on Day 1, followed by BID dosing from Day 2 to 14 . There was a 2-week washout period between each treatment period and a 12 - 14-day follow-up period. Patients were resident in the research facility from Day -1 to Day 2 and from Day 13 to Day 15 of each treatment period.

\section{Drug formulation and administration}

Ezutromid was administered as an aqueous microfluidized suspension; placebo was supplied in a matching formulation. All doses were administered in a blinded fashion (via oral syringe) and were to be taken immediately following a healthy balanced meal and followed consecutively with $100 \mathrm{~mL}$ full fat milk. Subjects were required to have the same meals on PK sampling days (Days 1 and 14 of each treatment period) where possible, i.e. the same breakfast, lunch, and dinner were to be provided to each patient.

At the Screening visit the patient's diet was assessed by the research dietician and dietary advice was provided to ensure the patient was eating a healthy balanced diet. It was recommended that patients consume 1200 1600 calories/day (age and activity dependent) with approximate proportions of fat (30\%), carbohydrate (45\%), and protein (25\%) incorporated into the diet. Consumption of foods from all major groups, micronutrients, and dietary fiber was encouraged. A parent/legal guardian recorded breakfast, lunch, dinner and snacks in a 3-day food diary (consecutive days including one weekend day) during each dosing and washout period. The research dietician continued to assess the patient's diet throughout the study.

\section{Assessment of pharmacokinetics}

Blood samples for the analysis of plasma concentrations of ezutromid and its major metabolites, dihydrodiol I and dihydrodiol III, were collected on Days 1 and 14 in each treatment period. Pharmacokinetic (PK) samples were collected at the following timepoints: On Day 1 single dose) at predose, $0.5,1,2.5,4,6,8,12$, and 24 
hours post-dose; and on Day 14 (bid dosing) at predose, $0.5,1,2.5,4,6,8$, and 10 hours relative to the morning (AM) dose and predose, $0.5,1,2.5,4,6,9$, and 12 hours relative to the evening (PM) dose.

A validated mass liquid chromatography with tandem mass spectrometry (LC-MS/MS) bioanalytical method was used to quantitate ezutromid in plasma with a lower limit of quantification (LLOQ) of $2 \mathrm{ng} / \mathrm{mL}$. An exploratory LC-MS/MS assay was used to measure plasma concentrations of dihydrodiol I and dihydrodiol III. Protein precipitation with LC-MS/MS was used for the determination of dihydrodiol I concentration; under LC conditions where both dihydrodiols I and III coelute, dihydrodiol III concentrations were determined from the difference between the resolved dihydrodiol I chromatographic peak and the chromatographic peak representing both metabolites. The LLOQ of both dihydrodiols was 0.5 or $1 \mathrm{ng} / \mathrm{mL}$, depending upon analytical run batch. The analytical measures were conducted by Covance Laboratories Ltd., Harrogate, UK. Additional details on analytical methods are provided in Appendix 1.

\section{Assessment of pharmacodynamics (PD)}

Blood samples were collected for measurement of the following PD: CPK and its isoenzymes, alanine aminotransferase (ALT) and aspartate aminotransferase (AST). The CPK isoenzymes assessed included CPKBB, CPK-MB, and CPK-MM; these different isoenzymes are located in different areas of the body, with CPKBB predominantly located in brain and lungs, CPK-MB predominantly located in the heart, and CPK-MM predominantly located in skeletal muscle Samples were collected during each treatment period prior to the morning dose on Days 1, 7, and 14. The analysis of PD biomarkers was performed by Covance Central Laboratory Service as part of the clinical laboratory evaluations.

\section{Assessment of Safety}

Safety was monitored throughout the study, with review of safety and PK prior to dose escalation; a final safety follow-up visit occurring 12 to 14 days after the final dose in treatment period 3. Triplicate supine ECGs were collected frequently throughout the study with more intensive collection when patients were resident

(Day 1 and Day 14 AM and PM: pre-dose, 0.5, 1, 4, 6 or $8 \mathrm{hr}$ post-dose). Adverse events (AE) and serious 
AE (SAE), physical exam, body weight, vital signs, and clinical laboratory parameters (blood and urine) were also recorded throughout the study.

\section{Pharmacokinetic and Statistical Analyses}

Pharmacokinetic analysis to derive non-compartmental parameters for ezutromid and its two major metabolites were conducted by Covance Early Clinical Biometrics using Phoenix WinNonlin ${ }^{\circledR}$ Version 6.2.1 (Pharsight Corporation). Parameters were determined for Day 1 (single dose) and Day 14 (AM and PM) (multiple dose) using actual sampling times. Values that were reported as below the LLOQ were set to zero (up to time of first quantifiable concentration), at the LLOQ (first instance after quantifiable concentrations) or "missing" (all other occurrences) for the calculation of parameters.

PK and safety data were summarized by treatment (1250 mg, $2500 \mathrm{mg}$, placebo) and day (where appropriate) using either descriptive statistics suitable for continuous data or counts and percentages for categorical data. Absolute values and changes from baseline were summarized by treatment group for each PD parameter (CPK, CPK isoenzymes, liver function tests [ALT and AST]). Data analysis was performed using SAS ${ }^{\circledR}$ Version 9.3.

\section{Results}

Twelve patients completed the study: four for each of the three treatment sequence groups. Baseline characteristics are shown in Table 1 and were similar across the three treatment groups. All patients were between 6.6 and 13.0 years old, with a mean age of 9.2 years. All patients were taking an oral corticosteroid for DMD prior to the study start. Additionally, two patients and one patient were on angiotensin converting enzyme inhibitors and a bisphosphonate, respectively, while eleven patients were on vitamin supplements.

\section{Pharmacokinetics of ezutromid}

Full pharmacokinetic profiles were available for most patients ( $\mathrm{n}=11$ on Day 14 following $2500 \mathrm{mg}$ ezutromid as one subject missed the Day 14 dose due to difficulties with cannulation). Ezutromid mean plasma 
concentration-time profiles for both dose levels on Day 1 and Day 14 (AM) are shown in Figure 2. PK parameters following single and multiple oral doses are summarized in Table 2.

Ezutromid exhibited prolonged absorption at both dose levels, with a secondary peak observed in some patients. Median $T_{\max }$ was approximately 2 to 4 hours post dose on Days 1 and 14; individual values ranged from 0.98 to 6.0 hours post-dose on Day 1 and 0.5 to 2 hours post AM or PM dose on Day 14. After reaching $\mathrm{C}_{\max }$, plasma concentrations of ezutromid declined in a generally biphasic manner, with resultant arithmetic mean terminal half-life of approximately 7 hours on Day 1 (range: 1.9 to 15.1 hours) as determined after single dose (10 patients each for 1250 and $2500 \mathrm{mg}$ ezutromid).

Exposure parameters $\left(\mathrm{AUC}_{\text {last }}, \mathrm{C}_{\max }\right.$ ) generally increased sub-proportionally with dose with a 1.2- to 1.5-fold increase for the 2-fold dose increase on both sampling days. However, between-patient variability at both dose levels was high with $\% \mathrm{CV} \geq 100 \%$ for most exposure parameters.

There was minimal evidence of accumulation of ezutromid upon twice daily dosing, with estimated accumulation ratios for $\mathrm{AUC}\left(\mathrm{AUC}_{0-\tau \text { - Day } 7} / \mathrm{AUC}_{0 \text {-tlast, Day 1) }}\right.$ of approximately 1 (1250 mg: 1.09, $2500 \mathrm{mg}$ : 0.94) but lower for $\mathrm{C}_{\max }(1250 \mathrm{mg}$ : 0.79, $2500 \mathrm{mg}$ : 0.72) (ratio of geometric least square means). There was marginal evidence of diurnal variation at steady state, with exposure to ezutromid $\left(\mathrm{C}_{\max }\right)$ being 1.1 and 1.4-fold greater for the evening dose compared to morning dose with the $1250 \mathrm{mg}$ and $2500 \mathrm{mg}$ doses, respectively.

\section{Pharmacokinetics of ezutromid metabolites}

The metabolite profiles (Figure 4) and relative exposures for dihydrodiol I (Table 3) and dihydrodiol III (Table 4) showed that dihydrodiol I was rapidly formed at both dose levels, and was observed in the plasma at approximately the same time $\left(\mathrm{T}_{\max }\right.$ and $\left.\mathrm{t}_{1 / 2}\right)$ as the parent drug. Dihydrodiol III was generated more slowly than dihydrodiol I, with median $\mathrm{T}_{\max }$ approximately 4 or 5 hours post-dose on Day 1 and Day 14 . For dihydrodiol I, for $\mathrm{AUC}_{0-\infty}$ (Day 1), $\mathrm{AUC}_{0-\tau}$ (Day 14), and $\mathrm{C}_{\max }$ (both days), the between-patient variability at the $1250 \mathrm{mg}$ and $2500 \mathrm{mg}$ dose levels was generally moderate with geometric CV\% ranging between 
approximately $23 \%$ and $47 \%$ on Day 1 and $29 \%$ to $44 \%$ on Day 14 . However, for dihydrodiol III, these values were generally high on Days 1 and Day 14, ranging between approximately $33 \%$ to $61 \%$ and $37 \%$ to $59 \%$, respectively. Both metabolites were more abundant than parent drug; on Day 14, the AUC metabolite to parent ratio (based on $\mathrm{AUC}_{0-\mathrm{t}}$ ) was approximately 18 and 40 for dihydrodiol I and III, respectively. However, there was minimal accumulation of either metabolite over time with accumulation ratios being approximately 1 for both metabolites at both dose levels.

\section{Pharmacodynamics}

No dose- or treatment-related trends were apparent for absolute or change from baseline values of CPK and its isoenzymes, ALT and AST at Day 7 and Day 14. At an individual level, there were no consistent increases or decreases from baseline in CPK, AST, or ALT in either the placebo, $1250 \mathrm{mg}$ ezutromid, or $2500 \mathrm{mg}$ ezutromid dose levels on Day 7 or Day 14.

\section{Safety}

The incidence of treatment-emergent AEs (TEAE) of all causalities following single and multiple oral doses of ezutromid is shown in Table 5. No severe AEs, no discontinuations due to an AE, no deaths, and no serious AEs were reported during the study. At both dose levels, the most frequently reported AEs were gastrointestinal disorders with pale stools $(100 \%, \mathrm{n}=12 / 12)$ being the most frequently reported, which were mild in severity and were resolved without treatment. The most frequently reported drug-related TEAE (including those considered by the Investigator to be related or possibly related to ezutromid) during the study was pale stools considered mild in severity. All TEAEs resolved following cessation of study treatment and without requiring concomitant medication. The other TEAEs, considered by the investigator to be possibly drug-related, were pain in the upper abdomen, nausea, and diarrhea which were reported by more than one patient following multiple oral doses. The reporting incidence of these TEAEs for patients administered active drug was similar to that reported during treatment with placebo. In the investigator's opinion, there were no 
clinically important findings in physical examination, vital signs, ECG changes or for clinical laboratory evaluations during this study.

\section{Discussion}

This clinical pharmacology study is one of a series of Phase 1 studies conducted with ezutromid to better understand the pharmacokinetics and safety of the molecule by improving the bioavailability for a new dosing regimen with a modified formulation, prior to embarking on a Phase 2 program.

The first reported Phase 1 study with ezutromid was in healthy volunteers where safety and pharmacokinetics were studied after single and repeat administration $(50-400 \mathrm{mg} / \mathrm{kg}) .{ }^{6}$ The data reported in the current study are generally consistent with this earlier study where ezutromid demonstrated rapid absorption and exposure increases were sub-proportional with dose. Additionally, a significant food effect had been demonstrated with increased exposure (approximately 4- to 5-fold increase in $\mathrm{AUC}_{0-\infty}$ and $\mathrm{C}_{\max }$ ) following a high fat breakfast at the $200 \mathrm{mg} / \mathrm{kg}$ dose when compared with fasted conditions (F3 formulation)

The second Phase 1 study, conducted in pediatric DMD patients, was also a single and multiple ascending dose study evaluating doses of $50 \mathrm{mg} / \mathrm{kg}$ BID, $100 \mathrm{mg} / \mathrm{kg}$ BID, and $100 \mathrm{mg} / \mathrm{kg}$ TID. ${ }^{7}$ The mean ideal body weight was estimated to be $25 \mathrm{~kg}$ (range 20 to $30 \mathrm{~kg}$ ). Using a start dose of $50 \mathrm{mg} / \mathrm{kg}$ this equated to $1250 \mathrm{mg}(50 \mathrm{mg}$ x $25 \mathrm{~kg}$ ) and for the $100 \mathrm{mg} / \mathrm{kg}$ cohort $2500 \mathrm{mg}(100 \mathrm{mg}$ x $25 \mathrm{~kg})$. These doses were therefore selected as the fixed doses for this study. Overall, exposure tended to be lower and much more variable than that observed in the first study with healthy volunteers. One potential reason for this difference could have been the lack of standardization of meals in the study, although patients were instructed to take the drug within 10 minutes of food.

The current study, therefore, was designed to assess whether optimizing the feeding conditions could increase the systemic exposure in DMD patients. Ezutromid is known to have low solubility and high permeability and undergoes extensive first pass metabolism leading to low oral bioavailability. In healthy volunteers, a positive impact of food had been observed and it was hypothesized that co-administration of ezutromid with foods containing fats, proteins, carbohydrates and soluble fiber could stimulate bile release in the human gut and 
convert the slowly dissolving ezutromid into a micellar solution or emulsion. The addition of full fat milk was hypothesized to further maximize this food effect. There was also the potential impact of reduced transit time, and therefore increased opportunity for absorption, by slowing gastric emptying with food. The results from the current study indicate that, using a combination of a balanced diet and administration of ezutromid with whole milk, systemic exposure can be improved in DMD patients. An exploratory comparison of exposure in six individuals who participated in both the prior (uncontrolled diet) DMD study and the current controlled diet study indicates that, in each case, exposure $\left(\mathrm{AUC}_{0-12}\right)$ was increased in the current study.

Following single and multiple oral administrations, the absorption profile of ezutromid was prolonged, manifesting as secondary peaks in some patients, which resulted in variable $t_{\max }$ at approximately 1.0 to 6.0 hours in all patients on Day 1 and 0.5 to 12.0 hours on Day 14 following the morning or evening dose. The underlying mechanism that causes the secondary peaks is not fully understood at this time. Ezutromid exhibited biphasic elimination with an apparent dose-independent terminal elimination half-life, with a mean $\mathrm{t}_{1 / 2}$ of approximately 7 hours on Day 1 , consistent with results from a previous clinical study in paediatric patients with DMD.

In vitro studies in human and DMD myoblasts and myotubes treated with ezutromid (in the presence of protein) followed a concentration-response curve with respect to utrophin protein with an estimated $\mathrm{EC}_{30}$ of $34 \mathrm{ng} / \mathrm{mL}(\sim 0.1 \mu \mathrm{M})$ and $\mathrm{EC}_{50}$ of $67 \mathrm{ng} / \mathrm{mL}(\sim 0.2 \mu \mathrm{M})$. In in-vivo efficacy studies, using dystrophin deficient $m d x$ mice, a single $50 \mathrm{mg} / \mathrm{kg}$ daily dose achieved levels above $30 \mathrm{ng} / \mathrm{mL}$ for several hours in a 24-hour period resulting in a significant reduction in the dystrophin muscle pathology. Therefore, the Phase 2 studies were designed to achieve exposures in excess of $30 \mathrm{ng} / \mathrm{mL}$. This study provides assurance that, with the formulation tested (F3) and instructions regarding food (balanced diet and whole fat milk), $2500 \mathrm{mg}$ BID ezutromid should achieve plasma concentrations that are expected to induce modulation of utrophin expression in future clinical trials. It should additionally be noted that metabolite exposures exceed those of parent molecule thus there is the potential for additional pharmacology to be driven by particularly dihydrodiol III. 
In both this study and previous ones in healthy volunteers ${ }^{6}$ and in pediatric patients, ${ }^{7}$ the most common AE was change in stool coloration (discolored or pale). In healthy volunteers, this AE occurred only at the higher doses of $200 \mathrm{mg} / \mathrm{kg}$ and $400 \mathrm{mg} / \mathrm{kg}$ and not $50 \mathrm{mg} / \mathrm{kg}$ and $100 \mathrm{mg} / \mathrm{kg}$, which led the authors to postulate that it was due to unabsorbed drug passing through the gastrointestinal tract. ${ }^{6}$ In the previous study in pediatric patients, of those treated with $50 \mathrm{mg} / \mathrm{kg}$ BID (n=4), $100 \mathrm{mg}$ BID ( $\mathrm{n}=4)$ and $100 \mathrm{mg} / \mathrm{kg}$ TID (n=4), one, three and three patients, respectively, experienced this AE. ${ }^{7}$ In the current study which used higher doses, twelve, eleven and nine patients at the $1250 \mathrm{mg}, 2500 \mathrm{mg}$ doses and placebo, respectively, had stool discoloration. As the placebo formulation had the same excipients as the active treatment, these findings suggest that the unabsorbed excipient (titanium dioxide at $5 \% \mathrm{w} / \mathrm{w}$ present only in the placebo) and unabsorbed ezutromid may be causing the stool discoloration. Other TEAEs, considered by the investigator to be possibly treatment related, following multiple oral doses of ezutromid, were pain in the upper abdomen, diarrhea, and nausea, although the incidence was similar in the placebo group. The first two of these were also reported in the previous pediatric study. ${ }^{7}$ Overall, ezutromid was considered safe and well-tolerated in pediatric DMD patients when administered at doses up to $2500 \mathrm{mg}$ BID.

In the previous open label Phase 1 study in pediatric patients, the majority of the patients showed reduced levels of serum CPK, ALT and AST levels compared with baseline levels over the 11 days of dosing. ${ }^{7}$ In the current study, there were no differences in change from baseline in plasma CPK, ALT, AST, or CPK isoenzymes following $1250 \mathrm{mg}$ or $2500 \mathrm{mg}$ BID ezutromid compared with placebo. These conflicting findings may be a consequence of differences in study design, wherein the current study was more tightly controlled (placebo-controlled, mandatory overnight stay to restrict activity) suggesting more reliable findings.

\section{Conclusions}

This study shows that ezutromid is safe and well tolerated at both single and multiple oral doses of 1250 and $2500 \mathrm{mg}$ BID in the pediatric DMD patients studied. Ezutromid systemic exposure in patients with DMD was enhanced by adherence to a balanced diet combined with guidance to consume a glass of full-fat milk with the drug. Considerable interpatient variability in exposure was observed, however, this study provides 
assurance that, with the formulation tested (F3) and instructions regarding food (balanced diet and whole fat milk), $2500 \mathrm{mg}$ BID ezutromid should achieve plasma concentrations capable of modulating utrophin expression in DMD boys in the phase 2 clinical trial. 


\section{Acknowledgements}

FM wishes to thank the support of the MRC Centre Grant and of the Muscular Dystrophy Campaign Centre Grant. He is also supported by the National Institute of Health Research Biomedical Research Center at Great Ormond Street Hospital for Children NHS Foundation Trust, and University College London. The views expressed are those of the author(s) and not necessarily those of the NHS, the NIHR or the Department of Health. The support of UtroDMD Alliance is also gratefully acknowledged. The authors would like to thank Neil Bhattacharya, Matt Anderton and Anne Heatherington (Summit Therapeutics Ltd) for input during the writing and review process. 


\section{References}

1. van Deutekom JC, van Ommen GJ. Advances in Duchenne muscular dystrophy gene therapy. Nat Rev Genet. 2003;4(10):774-783.

2. Bushby K, Finkel R, Birnkrant DJ, et al. Diagnosis and management of Duchenne muscular dystrophy, part 1: diagnosis, and pharmacological and psychosocial management. Lancet Neurol. 2010;9(1):77-93.

3. Miura P, Jasmin BJ. Utrophin upregulation for treating Duchenne or Becker muscular dystrophy: how close are we? Trends Mol Med. 2006;12(3):122-129.

4. Chancellor DR, Davies KE, De Moor O, et al. Discovery of 2-arylbenzoxazoles as upregulators of utrophin production for the treatment of Duchenne muscular dystrophy. J Med Chem. 2011;54(9):3241-3250.

5. Tinsley JM, Fairclough RJ, Storer R, et al. Daily treatment with SMTC1100, a novel small molecule utrophin upregulator, dramatically reduces the dystrophic symptoms in the mdx mouse. PLoS One. 2011;6(5):e19189.

6. Tinsley J, Robinson N, Davies KE. Safety, tolerability, and pharmacokinetics of SMT C1100, a 2arylbenzoxazole utrophin modulator, following single- and multiple-dose administration to healthy male adult volunteers. J Clin Pharmacol. 2015;55(6):698-707.

7. Ricotti V, Spinty S, Roper H, et al. Safety, Tolerability, and Pharmacokinetics of SMT C1100, a 2Arylbenzoxazole Utrophin Modulator, following Single- and Multiple-Dose Administration to Pediatric Patients with Duchenne Muscular Dystrophy. PLoS One. 2016;11(4):e0152840. 


\section{Appendix 1: Bioanalytical Details}

A validated mass liquid chromatography with tandem mass spectrometry (LC-MS/MS) bioanalytical method was used to quantitate ezutromid in plasma. Samples were extracted with acetonitrile and analyzed using a Waters Acquity UPLC system connected to a Sciex API 4000 mass spectrometer. A BEH column (C18, 1.7 $\mu \mathrm{m}, 2.1 \times 50 \mathrm{~mm})$ was used, and a gradient programmed from $40 \%$ mobile phase $\mathrm{A}(0.1 \%$ formic acid $)$ and $60 \%$ mobile phase $\mathrm{B}(0.1 \%$ formic acid in methanol) to $10 \% \mathrm{~A}-90 \% \mathrm{~B}$, with a flow rate of $0.5 \mathrm{~mL} / \mathrm{min}$. MS/MS was operated in Turbo IonSpray (positive ion) mode, and the mass transition monitored was $\mathrm{m} / \mathrm{z}$ 338.2 to 244.8 ( $\pm 0.5 \mathrm{Da}$ ) for ezutromid ( $\mathrm{m} / \mathrm{z} 341.1$ to 244.8 for internal standard). The lower limit of quantification (LLOQ) was $2 \mathrm{ng} / \mathrm{mL}$, with linearity demonstrable up to $2000 \mathrm{ng} / \mathrm{mL}$. During validation, mean intra- and inter-assay precision values were $\leq 6.9$ and $\leq 7.7 \% \mathrm{CV}$, respectively. The accuracy values across the calibration range were 93.3 to $106.4 \%$. The mean recovery of ezutromid from plasma ranged from 96.2 to $97.8 \%$. The internal standard was SMT18717 (which is structurally identical to ezutromid except that three hydrogen atoms are replaced by deuterium atoms) which had a mean recovery from plasma of $97.8 \%$. During sample analysis, all samples were run in accordance with the validated method and Covance SOPs.

An exploratory LC-MS/MS assay was used to measure plasma concentrations of dihydrodiol I and dihydrodiol III. Samples were extracted with acetonitrile and analyzed using a Waters Acquity UPLC system connected to a Sciex API 5500 mass spectrometer. A BEH column $(\mathrm{C} 18,1.7 \mu \mathrm{m}, 2.1$ x $50 \mathrm{~mm})$ was used, with mobile phase A comprising water/formic acid (100:0.2 v/v) and mobile phase B methanol/formic acid (100:0.2 v/v). The concentration of dihydrodiol I was determined under chromatographic conditions that resolved dihydrodiol I, dihydrodiol III, and ezutromid from each other (gradient from $40 \% \mathrm{~B}$ to $98 \% \mathrm{~B}$, total run time 8.5 minutes). MS/MS was operated in Turbo IonSpray (positive ion) mode, and the mass transition monitored was $\mathrm{m} / z 372.15$ to 279.45 for dihydrodiol I ( $\mathrm{m} / z$ 341.10 to 244.80 for SMT18717 internal standard). The method was qualified, but not validated; across all batches, the LLOQ was 0.5 or $1 \mathrm{ng} / \mathrm{mL}$, linear up to $500 \mathrm{ng} / \mathrm{mL}$, and $\%$ bias for quality control samples ranged from -13.9 to $3.4 \%$. Insufficient dihydrodiol III was available to prepare calibration and quality 
control standards. The concentration of dihydrodiol III was determined using a fast gradient (from $30 \%$ B to $98 \% \mathrm{~B}$, total run time 3.5 minutes) that forced coelution of the dihydrodiol I and dihydrodiol III peaks. MS/MS conditions were as for dihydrodiol I quantification, and the dihydrodiol III concentrations were calculated based on the difference in back-calculated values for the combined and resolved chromatographic peaks. No acceptance criteria were set for qualification of the method; across all batches, the LLOQ was 0.5 or $1 \mathrm{ng} / \mathrm{mL}$, linear up to $500 \mathrm{ng} / \mathrm{mL}$, and \% bias for quality control samples ranged from -12.7 to $21.8 \%$. 